\title{
Apropiación de dispositivos móviles en educación: una experiencia de sistematización sobre uso pedagógico de TIC en tabletas
}

\author{
Clara Esperanza Pedraza Goyeneche ${ }^{1}$ \\ Olinda Flor Amado Plata ${ }^{2}$ \\ Pablo Alexander Munévar García ${ }^{3}$
}

Recibido: 13-06-2019

Aceptado: 23-08-2019

\section{Resumen}

El presente artículo da cuenta de la experiencia de sistematización sobre un proyecto de uso pedagógico de tecnologías de la información y la comunicación (TIC) con tabletas en el municipio de Pauna, departamento de Boyacá, Colombia. El alcance de dicha propuesta consistió en abordar, desde una perspectiva de corte pedagógico, didáctico y tecnológico, la pertinencia de apropiar los dispositivos móviles en la educación básica y media, en particular las tablets o tabletas. Dicho alcance permitió diseñar una estrategia

\footnotetext{
1. Licenciada en Psicopedagogía. Especialista en Diseño de Ambientes de Aprendizaje. Magíster en Administración Educativa y Doctoranda en Estudios Interdisciplinares de Género. Decana de la Escuela de Ciencias de la Educación de la Universidad Nacional Abierta y a Distancia (UNAD), Colombia.

ORCID: http://orcid.org/0000-0002-0150-5399

Correo electrónico: clara.pedraza@unad.edu.co
}

2. Psicóloga. Especialista de Orientación de Procesos de Formación Diseño y Desarrollo Curricular. Especialista en Gestión de Empresas. Especialista en Gerencia de los Servicios de Salud. Magíster en Docencia de la Educación Superior. Doctora en Gerencia y Política Educativa. Equipo académico del Programa Formación de Formadores. Docente de la Escuela de Ciencias de la Educación de la Universidad Nacional Abierta y a Distancia (UNAD), Colombia. ORCID: https://orcid.org/0000-0003-2740-223X

Correo electrónico: olinda.amado@unad.edu.co

3. Licenciado en Electrónica. Magíster en Educación. Doctor en Educación, currículo, profesorado e instituciones educativas. Equipo académico del Programa Formador de Formadores. Líder grupo de investigación Ubuntu. Docente Asociado de la Escuela de Ciencias de la Educación de la Universidad Nacional Abierta y a Distancia (UNAD), Colombia.

ORCID: https://orcid.org/0000-0001-9577-9253

Correo electrónico: pablo.munevar@unad.edu.co 
de formación en m-learning que incluyó a docentes, estudiantes y padres de familia desde una perspectiva crítica, innovadora y creativa, bajo el Aprendizaje Basado en Proyectos (ABP), como método que establece la ruta orientadora que trazó la estrategia de formación.

Palabras clave: Aprendizaje móvil, TIC, formación de docentes, apropiación tecnológica Aprendizaje Basado en Proyectos (ABP).

\section{Use of mobile devices in education: a systematization experience on pedagogical use of ICT (tablets)}

\section{Abstract}

This article gives an account of a pedagogical project's systematization experience using information and communication technologies (ICT) with tablets in the municipality of Pauna, department of Boyacá, Colombia. This proposal aimed to address, from a pedagogical, didactic and technological perspective, the relevance of using mobile devices in basic and secondary education, in particular tablets. This scope allowed the design of an $m$-learning strategy that included teachers, students and parents from a critical, innovative and creative perspective, under ProjectBased Learning (PBL).

Keywords: mobile learning, ICT, teacher training, technological use, Project Based Learning (PBL). 


\section{Introducción}

Los elementos que fundamentan la presente propuesta se articulan entre las siguientes categorías conceptuales: $m$-learning; apropiación de las tecnologías de la información y la comunicación (TIC) en la educación, la formación de maestros con dispositivos móviles, y el Aprendizaje Basado en Proyectos (ABP), todas ellas fundamentales para la estrategia pedagógica.

\section{El mobile learning}

El mobile learning (también llamado m-learning) se concibe como una metodología de trabajo que emerge desde la incorporación de los dispositivos móviles en la educación con fines de potenciar el aprendizaje. Para la Organización de las Naciones Unidas para la Educación, la Ciencia y la Cultura (Unesco), este tipo de aprendizaje se define así:

El aprendizaje móvil, personalizado, portátil, cooperativo, interactivo y ubicado en el contexto, presenta características singulares que no posee el aprendizaje tradicional mediante el uso de instrumentos electrónicos (e-learning). En el primero se hace hincapié en el acceso al conocimiento en el momento adecuado, ya que por su conducto la instrucción puede realizarse en cualquier lugar y en todo momento. Por eso, en tanto que dispositivo de ayuda al aprendizaje formal e informal, posee un enorme potencial para transformar las prestaciones educativas y la capacitación. (West \& Vosloo, 2013, p. 7)

Por tanto, la perspectiva actual y contemporánea de la apropiación de los dispositivos móviles en la educación trasciende de una perspectiva basada en el uso de la tecnología a un enfoque más de apropiación. Esto se debe al hecho de acceder a la información $y$ al conocimiento en cualquier lugar con el uso de diversas he- 
rramientas tecnológicas (celulares, tabletas, smartphones, personal digital assistant (PDA), entre otros) y con la masificación del acceso a Internet, que garantiza un acceso a la nube con el solo hecho de contar con una conexión libre —en algunos lugares con wifi abierto- o paga.

Esta dinámica permite legitimar la presencia de teorías del aprendizaje como las basadas en el conectivismo y el aprendizaje ubicuo, entre otros. El hecho de contar con contenidos de acceso a la nube y otros almacenados dentro de los mismos dispositivos por medio de aplicaciones (apps) permite e incentiva en los estudiantes un uso pedagógico, vivencial interactivo de las TIC, con las diversas utilidades de corte tecnológico que ofrecen en su interior dichos artefactos, proporcionando una oportunidad pedagógica enfocada en el aprendizaje.

\section{Apropiación de las TIC en la educación}

Trascender de la perspectiva de uso a la apropiación de las TIC permite incorporar las tecnologías en la educación, más allá de una perspectiva instrumental, y enfocarla en lo cognitivo. Es decir, prima lo relacionado con el sentido y significado de las TIC, que su abordaje utilitario. La solución de problemas, la simulación de escenarios, la comprensión de los fenómenos y la realización de proyectos son algunos aspectos que las TIC deben fomentar en su implementación, y no sesgarse al uso de la herramienta.

Alberts (2013) plantea que los modelos de apropiación tecnológica representan una de las "tres principales líneas de pensamiento dentro de la investigación en Sistemas de Información, en los cuales la tesis principal es que la apropiación cambia el significado atribuido a la tecnología, así como las formas de acción en que la utilizamos" (p. 35). La apropiación conduce a un nivel de interiorización y de subjetividad que se moldea y se configura en una mayor aproximación al objeto. Es decir, cuanto mayor sentido y significado le otorgue el objeto al sujeto, tanto mayor será el nivel de apropiación. 
Crovi-Druetta y López-González (2011, citado por Morales, Lavigne, \& Mercado, 2016) definen los siguientes niveles de apropiación de las TIC:

- Acceso. Está vinculado con la posibilidad de ofrecer recursos para todos los usuarios: es el punto de partida para la apropiación. Incluye dos categorías: acceso a la infraestructura y acceso a la alfabetización digital formal (cursos curriculares y extracurriculares).

- Uso. Vinculado al uso cotidiano de las TIC y el aprovechamiento máximo de éstas al realizar ciertas actividades. Está condicionado por el acceso y va desde un grado de uso esporádico a uno de uso intensivo.

- Apropiación. La apropiación tiene lugar en un contexto socio-histórico particular en el cual el individuo además de tener acceso a las TIC, cuenta con habilidades para utilizarlas, de manera que, cobran tal relevancia en sus actividades cotidianas, que son integradas a las prácticas sociales. (p. 3)

Por lo tanto, en un escenario formativo diverso, como los que se encuentran en los entornos escolares, se debe dar un mayor sentido del valor del objeto tecnológico como mediador del aprendizaje, desde el grado de valor de apropiación que le significa al estudiante para desarrollar a plenitud su cotidianidad y el desarrollo de sus competencias para la vida.

\section{La formación de maestros con dispositivos móviles}

Muchas experiencias documentadas a nivel internacional, mundialmente reconocidas, coinciden en afirmar que el m-learning y, en particular, las propuestas desarrolladas con smartphones y tabletas 
han incidido en el desarrollo de nuevas alternativas que potencian el aprendizaje y permiten innovar didáctica y pedagógicamente las prácticas docentes en el aula y fuera de ella.

Proyectos como los realizados en México (2013), Chile (2014), Estados Unidos (2014), Argentina (2014)4, entre otros, dan cuenta de las bondades y beneficios del desarrollo de estrategias de incorporación de dispositivos móviles en la escuela, donde el papel fundamental de formación de los docentes y la participación de los estudiantes motiva aspectos asociados a la innovación y la creatividad.

En este sentido, recobra valor lo planteado por Coll y Monereo (2007), en lo que compete a la innovación desde la perspectiva de los docentes:

Cuando somos capaces de elaborar una representación personal sobre un objeto de la realidad o concepto que pretendemos aprender. Esa elaboración implica aproximarse a dicho objeto o contenido con la finalidad de aprehenderlo; no se trata de una aproximación vacía, sino desde las experiencias, intereses o conocimientos previos que se presumiblemente pueden dar cuenta de la novedad...En ese proceso, no sólo modificamos lo que ya poseíamos, sino que también interpretamos lo nuevo de forma peculiar, de manera que podamos integrarlo y hacerlo nuestro. (p. 13)

Por esta razón, desde la perspectiva de los maestros, las propuestas deben, más allá de incorporar un conjunto de recursos y

4. Se resaltan experiencias como los trabajos realizados por Castillo-Santos y Rivera-Castañeda (2014), quienes hacen un estudio comparativo en la Ciudad de México y Campeche, en el que se destaca el uso del $m$-learning como un escenario de construcción de conocimiento entre docentes y estudiantes. Para el caso chileno, en 2014 la Fundación Telefónica orientó con éxito un proceso formativo con estudiantes de niveles básico y medio de educación, con un alto impacto de aceptación, mientras que en Argentina en el mismo año el proyecto Educ.ar sistematizó experiencias exitosas del modelo 1 a 1, incursionando a dispositivos móviles en la escuela. Asimismo, se destaca en Estados Unidos el proyecto liderado por Muñoz-Reyes (2014) del Programa Seeds of Empowerment de la Escuela de Educación de la Universidad de Stanford, con el proyecto Smile (Mobile Inquiry based Learning Environment). 
herramientas, apuntar a diseñar escenarios diversos, creativos en innovadores que permitan resignificar actividades de aprendizaje desde la apropiación de estos recursos educativos en el aula.

\section{Aprendizaje Basado en Proyectos}

En la estrategia del Aprendizaje Basado en Proyectos (ABP), el docente es un creador que proporciona una experiencia de aprendizaje al estudiante a través de una propuesta significativa sobre problemas contextuales, con el fin de desarrollar integralmente las capacidades, habilidades, actitudes y valores de sus estudiantes con respecto al entorno social en el que este se desenvuelve. De acuerdo con Restrepo et al. (2013), emplear una estrategia didáctica de ABP ofrece las siguientes ventajas:

(a) la metodología de proyectos es una estrategia para el aprendizaje que permite el logro de aprendizajes significativos, porque surgen de actividades relevantes para los estudiantes, y contemplan muchas veces objetivos y contenidos que van más allá que los curriculares. (b) Permite la integración de asignaturas, reforzando la visión de conjunto de los saberes humanos. (c) Permite organizar actividades en torno a un fin común, definido por los intereses de los estudiantes y con el compromiso adquirido por ellos. (d) Fomenta la creatividad, la responsabilidad individual, el trabajo colaborativo y la capacidad crítica, entre otros. (p. 22)

De este modo, el ABP se orienta hacia el desarrollo de un proyecto o plan siguiendo el enfoque de diseño de proyectos. Así, las actividades se orientan a la planeación de la solución de un problema complejo; el trabajo se lleva a cabo en grupos; los estudiantes tienen mayor autonomía que en una clase tradicional y hacen uso de diversos recursos (Restrepo et al., 2013). 


\section{Metodología}

La presente propuesta se desarrolla en Pauna, municipio del departamento de Boyacá, ubicado a $176 \mathrm{~km}$ de Bogotá. Como parte de la estrategia Tabletas para Educar del Ministerio de las Tecnologías de la Información y la Comunicación (MinTIC), se genera un convenio de cooperación entre ese ministerio y la Universidad Nacional Abierta y a Distancia (UNAD), en el que se otorga a la institución la labor de formar a los docentes, estudiantes, directivos y padres de familia que pertenecen a la comunidad académica del municipio.

La estrategia tiene como propósito incentivar el uso pedagógico de dichas tabletas en los escenarios escolares, para lo cual se establece una serie de fases que involucraron a los actores que tuvieron un papel protagonista en el desarrollo de la propuesta. Para ello, se generaron cinco fases de desarrollo de la propuesta que se presenta a continuación.

\section{Fase de caracterización}

Gracias a la gestión de la Secretaría de Desarrollo Social de Pauna, se hace el contacto para interactuar con los 51 docentes y tres directivos docentes de las instituciones donde se desarrolló la propuesta; allí, el equipo Formación de Formadores de la UNAD desarrolla estrategias de caracterización de dicha población. Se realizó un primer acercamiento, a través de un sistema de videoconferencia con la población involucrada. En dicho acercamiento se implementó un instrumento de caracterización diagnóstica de los docentes.

\section{Fase de formación}

El equipo del programa Formación de Formadores de la UNAD diseña un diplomado denominado "Uso pedagógico de las TIC en tabletas", con una duración de 96 horas o el equivalente a dos 
créditos académicos. Esto permitió garantizar la inscripción de los 51 maestros, quienes a su vez fueron acompañados de forma presencial, junto con la formación de estudiantes, directivos, padres de familia y el semillero de investigación, conformado también por los estudiantes.

Asimismo, para reforzar y fortalecer el proceso académico en el acompañamiento presencial en contexto, se desarrolló una serie de talleres formativos que permitieron conocer los beneficios didácticos, pedagógicos y prácticos que brinda la estrategia educativa Tabletas para Educar. De este modo, se genera un espacio para el uso pertinente del dispositivo digital, con el fin de dinamizar y fortalecerlos procesos de enseñanza-aprendizaje mediante la apropiación de TIC a través de la estrategia metodológica y la investigación y construcción de proyectos de aula.

Cabe resaltar que los estudiantes que participaron como semillero, en particular el grupo de la Institución Educativa Técnico Nacionalizado de Pauna, tuvieron la experiencia de instalar apps o aplicaciones educativas en las tabletas, que les permitió fortalecer niveles de aprendizaje; de manera que estas se convirtieron en herramientas mediadoras en su formación, para potenciar el aprendizaje significativo para toda la vida.

\section{Fase de formación en campus virtual}

La estrategia se encuentra dividida en tres fases que sitúan al estudiante en una problemática contextual que se pretende abordar mediante la estructuración y el desarrollo de un Proyecto de Aula. Se inicia con una primera fase, llamada "Proyecto de Aula y manejo $\mathrm{TIC}^{\prime}$; la fase dos se denomina "Aplicaciones educativas en Tabletas", y se concluye con una tercera fase llamada "Desarrollo del proyecto de aula: Incorporando tecnología".

Dicha formación se realizó directamente desde el campus virtual de la UNAD, cuyo curso se enfocó en el desarrollo de competencias de uso y apropiación de tecnologías móviles, por medio de un 
Ambiente Virtual de Aprendizaje (figura 1), cuyos entornos fueron diagramados desde una perspectiva amigable para la formación de la población objetivo.


Fafase 1: Aprendizaje Basado en Probblemas (ABPr) y Compentencias Tic

Cocnologia y ambientes de aprendizaje

EäBibliografia Complementaria, Fase 1

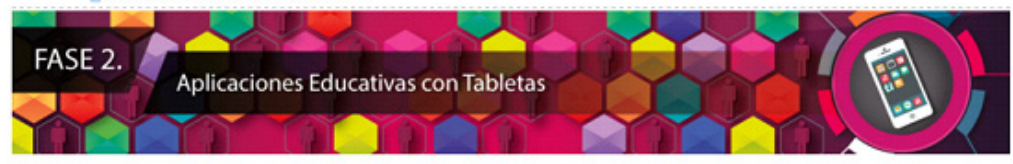

Gafase 2: Estructuraciôn del proyecto de aula

C-Experiencias: Aplicaciones educativas en Tabletas

Dibiografia complementaria, Fase 2

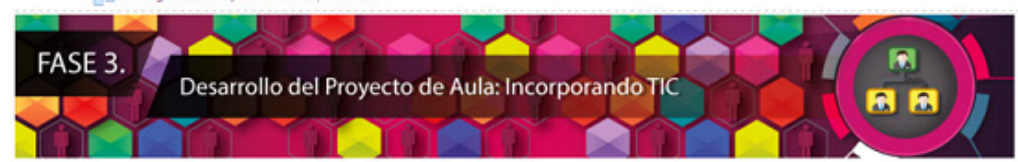

Figura 1. Elementos visuales de Aula Virtual del Diplomado de Uso pedagógico de TIC en tabletas. Fuente: elaboración propia. 


\section{Fase de formación in-situ}

Con la formación virtual, paralelamente se realizó un proceso de acompañamiento al desarrollo de la estrategia, que permitió, entre otros aspectos, la sensibilización y el acercamiento a la población y, por supuesto, el desarrollo de las actividades y proyectos, así como la formación misma de los estudiantes, docentes, directivos y padres de familia.

\section{Fase de innovación}

Bajo la metodología del ABP, y como producto de la fase formativa del diplomado, los actores involucrados en la estrategia llegaron al diseño de proyectos tecnopedagógicos, caracterizados por un rigor y una perspectiva de apropiación de las TIC con el uso de las tabletas, reconociendo las necesidades y realidades de sus regiones y su institución.

\section{Resultados}

Fundamentalmente, la estrategia cumplió con los objetivos propuestos, a través de la formación de 51 docentes, certificando con el diplomado a 29 de ellos, así como la formación de 1.100 niños y a 400 padres de familia. Asimismo, se logró una formación de un semillero de 40 estudiantes quienes, además de liderar el proceso de la mano con sus maestros en la dinamización y apropiación de las tabletas con un sentido didáctico y pedagógico, conciben las TIC desde una perspectiva social y creativa. Estos resultados se pueden ver sintetizados en la figura 2. 


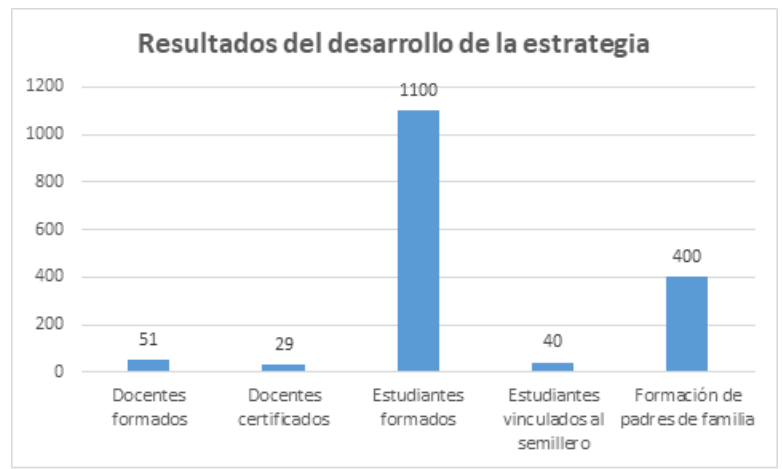

Figura 2. Resultados del desarrollo de la estrategia de acompañamiento al Programa Tabletas para Educar en 4 sedes de las instituciones del municipio de Pauna - Boyacá. Fuente: autores (2016).

\section{Los proyectos de aula desarrollados por las Instituciones Educa- tivas en el municipio de Pauna, Boyacá, que fueron acompañados y asesorados por la UNAD desde el Programa Formación de Forma- dores, se presentan en la tabla 1.}

Tabla 1. Proyectos de aulas desarrollados en la estrategia tabletas para educar.

\begin{tabular}{|c|c|}
\hline $\begin{array}{l}\text { Nombre del proyecto } \\
\text { de aula }\end{array}$ & Descripción \\
\hline Las TIC más allá del papel & $\begin{array}{c}\text { En la sede Institución Educativa Concentración Alianza para el Progreso (Alpro), } \\
\text { se desarrolló el proyecto de aula "Las TIC más allá del papel", mediante la } \\
\text { implementación de una página institucional con el Recurso Educativo Digital } \\
\text { desarrollado bajo la herramienta de uso libre Wix. Este espacio le permite a cada } \\
\text { docente acceder para vincular las diferentes actividades y demás proyectos de } \\
\text { la comunidad académica, así como socializarlos para enriquecer el desarrollo del } \\
\text { Proyecto Educativo Institucional (PEI) de manera interdisciplinaria, vinculando } \\
\text { todas las asignaturas. En este sentido, las TIC, a través del proceso académico } \\
\text { Uso pedagógico de las TIC en tabletas, permite llegar a estudiantes, docentes, } \\
\text { directivos y padres de familia con iniciativas didáctico-pedagógicas innovadoras } \\
\text { y emergentes, que dinamizan y fortalecen los procesos de enseñanza- } \\
\text { aprendizaje. }\end{array}$ \\
\hline $\begin{array}{l}\text { Fortalecimiento de las } \\
\text { competencias en inglés } \\
\text { a través del uso de las } \\
\text { tabletas }\end{array}$ & $\begin{array}{l}\text { Los docentes de la Institución Educativa Técnico Nacionalizado de Pauna } \\
\text { desarrollaron el proyecto de aula enfocado en el fortalecimiento de competencias } \\
\text { en inglés, vinculando las tabletas como herramienta de apoyo y de formación } \\
\text { pedagógica. }\end{array}$ \\
\hline $\begin{array}{l}\text { Uso adecuado del tiempo } \\
\text { libre mediante el uso de } \\
\text { tabletas }\end{array}$ & $\begin{array}{l}\text { La Institución educativa Técnico Agropecuario de Desarrollo Rural, con su } \\
\text { equipo de trabajo conformado por docentes, estudiantes, directivos y padres } \\
\text { de familia, desarrolló este proyecto de aula, en el que la dinámica evidenciada } \\
\text { tiene especial relevancia, ya que los estudiantes de grado once apoyaron } \\
\text { la capacitación de los niños y las niñas de básica primaria, utilizando las } \\
\text { tabletas como herramienta dinamizadora del aprendizaje, en las que instalaron } \\
\text { juegos relacionados con el tipo de pensamiento lógico-matemático, con el fin } \\
\text { de desarrollarlo desde diversas actividades aplicables en los ambientes de } \\
\text { aprendizaje. }\end{array}$ \\
\hline
\end{tabular}




\begin{tabular}{|c|c|}
\hline $\begin{array}{c}\text { Nombre del proyecto } \\
\text { de aula }\end{array}$ & Descripción \\
\hline Pioneros en saberes & $\begin{array}{c}\text { La Institución Educativa Monte y Pinal desarrolló este proyecto de aula, que tuvo } \\
\text { como propósito propender por que los estudiantes se cuestionen y se inquieten } \\
\text { por el mundo de las Ciencias a través de la tecnología. En este, las tabletas } \\
\text { tienen un significado relevante, ya que facilitan al estudiante la investigación } \\
\text { y la creación a partir de sus propias iniciativas de vida y las orientaciones del } \\
\text { maestro, con quien pueden compartir experiencias reales que documentan a } \\
\text { través del dispositivo digital, incluso como biblioteca y Play Store, entre otras } \\
\text { formas que le permiten reforzar el aprendizaje en las diferentes asignaturas. De } \\
\text { este modo, participan individual y grupalmente para socializar experiencias y } \\
\text { realimentarse como comunidad educativa. }\end{array}$ \\
\hline
\end{tabular}

Fuente: elaboración propia.

En consecuencia, se desarrollaron cuatro proyectos tecnopedagógicos de aula, en los que la Institución Educativa Técnico Agropecuario de Desarrollo Regional (con sus sedes Central y Monte y Pinal) y la Institución Educativa Técnico Nacionalizado de Pauna (con sus sedes Central y Alpro) generaron propuestas de innovación que se caracterizaron por un amplio sentido del liderazgo de los maestros a cargo, que fueron socializados con éxito (figura 3). Sin duda, el papel de los estudiantes y padres de familia en el desarrollo del proceso permitió incorporar aspectos de empoderamiento de las tecnologías con fines formativos.






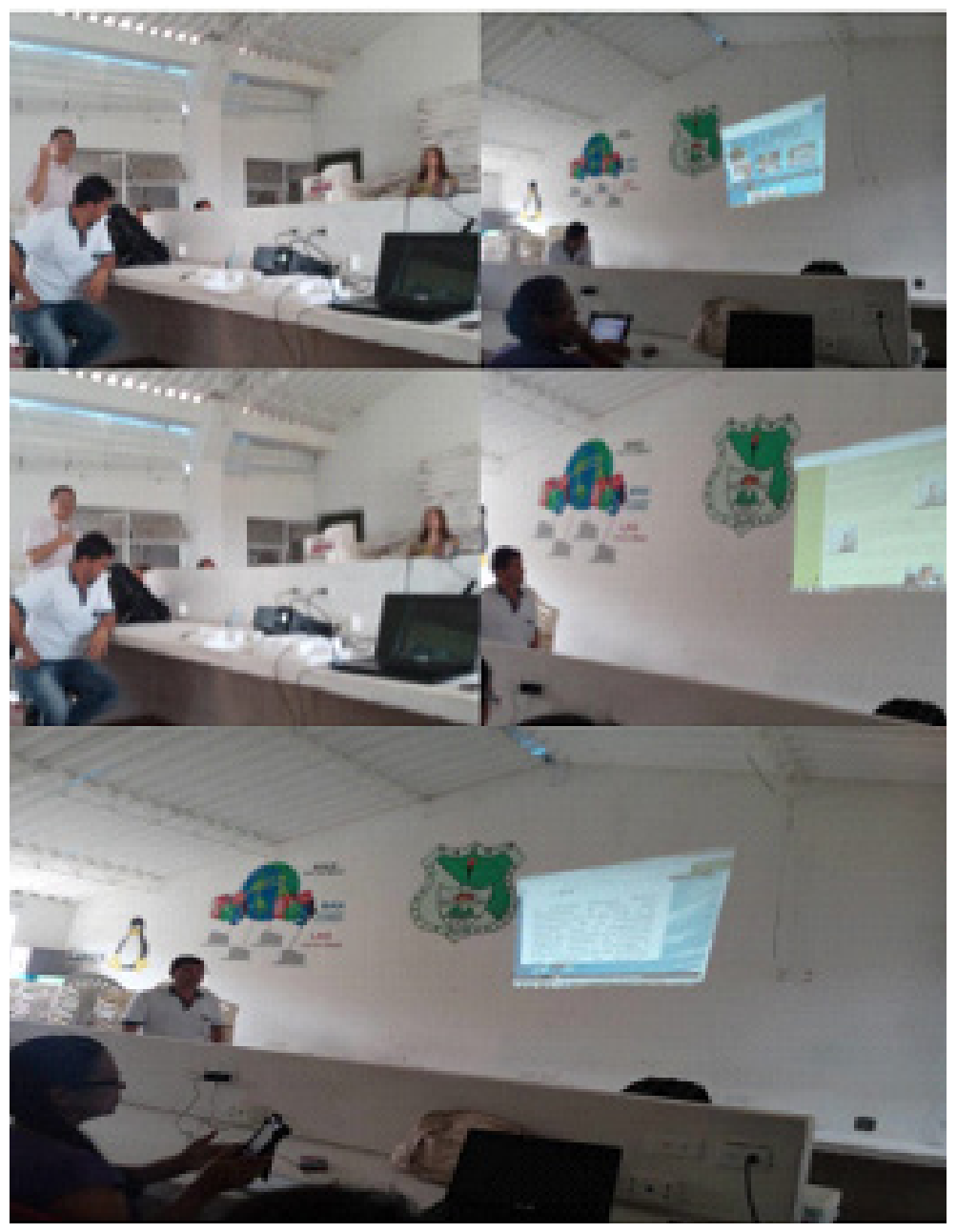

Figura 3. Socialización de proyectos, resultado de la estrategia de acompañamiento en el diplomado "Uso pedagógico de las TIC en tabletas". Fuente: propiedad de los autores.

Desde la dinámica de los proyectos de aula, se innovó en el uso del recurso educativo digital bajo la herramienta Wix sistematizando la experiencia (figura 4). Finalmente, los resultados del proceso académico "Uso de las TIC a través de tabletas" fueron almacenados y puestos a disposición de las cuatro Instituciones Educativas, con el fin de que la comunidad educativa pudiera acceder y conocer su experiencia. 




Figura 4. Evidencia del Recurso Educativo Digital diseñado para documentar el proceso académico del curso "Uso pedagógico de las TIC en tabletas". Fuente: propiedad de los autores.

Además, a partir de la experiencia académica se editó una cartiIla digital contenida en cinco unidades.

- La primera unidad tiene como propósito exponer qué es una tableta, cuáles son sus accesorios, cuál es su sistema operativo, qué son las aplicaciones o apps y cuáles tienen utilidad para la educación, además de mostrar políticas para su uso en ambientes de aprendizaje, consideraciones sobre su cuidado y recomendaciones relacionadas con la seguridad de uso básico.

- La segunda unidad aborda aspectos relacionados con el uso de la tableta, el control parental y cómo facilitar su uso para personas con dificultades perceptivas.

- La unidad tres se concentra en la presencia de las tabletas en entornos educativos; describe la importancia del aprendizaje táctil debido a las características de interacción de la tableta; muestra los enfoques favorecedores del aprendizaje con tabletas, y sus aportes y ventajas para el entorno educativo en el aula. 
- La unidad cuatro detalla el convenio de cooperación desarrollado y describe las experiencias adelantadas en las Instituciones Educativas objeto del convenio.

- Finalmente, la unidad cinco describe experiencias en el desarrollo de los proyectos tecnopedagógicos de aula en las cuatro Instituciones Educativas objeto del convenio de cooperación interinstitucional.

\section{Conclusiones}

Sin lugar a dudas, los dispositivos móviles son un recurso pedagógico que potencian el aprendizaje, disminuyen la brecha digital y acercan al estudiantado y a los docentes al conocimiento. Las experiencias de aula presentadas dan cuenta del proceso formativo, que se logró con el objeto del convenio, además de la formación de los maestros, los estudiantes y la comunidad educativa en general, junto con el semillero de estudiantes. Así, la generación de proyectos se concibe como una propuesta de continuidad y de aplicación de forma permanente en estas instituciones.

El diplomado realizado en el marco del proyecto fue un escenario que brindó herramientas tanto desde el ámbito virtual como en actividades in situ, con el fin de fomentar estrategias tecnopedagógicas basadas en proyectos. Los docentes, a través de la estrategia tabletas para educar, realizaron proyectos tecnopedagógicos de aula que facilitaron en los estudiantes el aprendizaje significativo para la vida.

Los proyectos y beneficios propiciados por este dispositivo móvil, a través de un aprendizaje autónomo y colaborativo, les permiten a los estudiantes apropiarse de las temáticas de una manera práctica y constructiva, obteniendo así un mejor desempeño académico y como personas, ya que encuentran en el proceso educativo una motivación real que fortalece su proyecto de vida. 
El desarrollo de esta metodología permitirá que los estudiantes y docentes adquirieran a través de las tabletas nuevos conocimientos, a partir del abordaje de un problema y el planteamiento de posibles alternativas de solución, en proyectos desarrollados en beneficio de la comunidad educativa y, en general, para el entorno en el que están inmersos los docentes y estudiantes.

Finalmente, se reconoce la propuesta de país a la que está dirigida la UNAD, como aporte a través de la Escuela Ciencias de la Educación en la formación de docentes en campos como la apropiación de tecnologías, llegando con propuestas desde una perspectiva pedagógica y didáctica de formación con tecnologías.

\section{Referencias bibliográficas}

Alberts, B. (2013). Technology Appropriation Revisited, Tesis de maestría, University of Twente, Enschede, Países Bajos. Recuperado de http:// essay.utwente.nl/62736/1/Berend_Alberts_-_BIT_Master_Thesis.pdf

Aparicio, O.Y. (2018). Las TIC como herramientas cognitivas para la investigación escolar. Revista Interamericana de Investigación, Educación y Pedagogía, RIIEP, 11(1). https://orcid.org/0000-0003-3535-6288

Aparicio, O.Y. (2018). El constructivismo y el construccionismo. Revista Interamericana de Investigación, Educación y Pedagogía, RIIEP, 11(2). https://orcid.org/0000-0003-3535-6288

Castillo-Santos, B., \& Rivera-Castañeda, M. (2014). El uso del mobile learning para favorecer la competencia referente al manejo de la información histórica y la socialización del conocimiento. Apertura, 6(2), 1-8. Recuperado de http://www.redalyc.org/pdf/688/68835725007.pdf

Coll, C., \& Monereo, C. (Eds.). (2008). Psicología de la Educación Virtual. Madrid: Morata. 
Konieczny, P. (2015). Lorenzo García Aretio: bases, mediaciones y futuro de la educación a distancia en la sociedad digital. Revista Interamericana de Investigación, Educación y Pedagogía, RIIEP, 8(1). DOI: https://doi. org/10.15332/s1657-107X.2015.0001.08

Morales, M., Lavigne, G. \& Mercado, M. A. (2016). Apropiación tecnológica de estudiantes rurales adscritos a una universidad virtual. Edutec, Revista Electrónica de Tecnología Educativa, 55, 1-13. Recuperado de http:// www.edutec.es/revista/index.php/edutec-e/article/download/645/ Edutec_n55_Morales_Lavigne_Mercado/

Muñoz-Reyes, C. (2014). Integrando la tecnología móvil y la pedagogía para un aprendizaje basado en la indagación. Recuperado de http:// www.relpe.org/integrando-la-tecnologia-movil-y-la-pedagogia-para-unaprendizaje-basado-en-la-indagacion/

Restrepo Álvarez, E., Gómez de Illera, M., Ahumada de la Rosa, V., Puentes Montaño, E., Meneses Parra, R., ... Nieto Gómez, L. (2013). Metodología, estrategias y herramientas didácticas para el diseño de cursos en ambientes virtuales de aprendizaje en la Universidad Abierta y a Distancia UNAD: estudio de caso como estrategia didáctica de aprendizaje. Recuperado de: https://repository.unad.edu.co/ handle/10596/5396

West, M., \& Vosloo, S. (2013). Directrices de la Unesco para las políticas de aprendizaje móvil. Recuperado de http://unesdoc.unesco.org/ images/0021/002196/219662S.pdf 